\title{
DISPERSÃO DE DIÁSPOROS NÃO MIRMECOCÓRICOS POR FORMIGAS: INFLUÊNCIA DO TIPO E ABUNDÂNCIA DO DIÁSPORO'
}

\begin{abstract}
Fernanda dos Santos Farnese ${ }^{2}$, Renata Bernardes Faria Campos ${ }^{2}$ e Gustavo Augusto Fonseca ${ }^{2}$
RESUMO - A mirmecocoria é importante processo para a dinâmica vegetacional em diferentes ecossistemas, principalmente na região neotropical. Entretanto, a relação entre formigas e diásporos não mirmecocóricos é pouco documentada em ambientes da América do Sul. Dessa forma, este estudo teve por objetivos quantificar a taxa de remoção de diásporos não mirmecocóricos em fragmento de Cerrado e verificar se a presença de recursos energéticos e, ou, a abundância dos diásporos são determinantes para sua remoção por formigas. Formigas removeram 19,81\% dos diásporos não mirmecocóricos coletados, e a presença de recursos energéticos influenciou significativamente essa remoção, não sendo significativa a relação entre abundância e remoção. Como a maioria dos diásporos coletados não era do tipo carnoso, com pouco ou nenhum recurso energético disponível para formigas, a importância da mirmecofauna para a remoção de diásporos não mirmecocóricos pode estar sendo subestimada.
\end{abstract}

Palavras-chave: Cerrado, Mirmecocoria e Elaiosomo.

\section{NON MYRMECOCHORUS DIASPORES DISPERSION BY ANTS: TYPE AND DIASPORE ABUNDANCE INFLUENCE}

\begin{abstract}
The myrmecochory is an important process for the vegetation dynamic in different ecosystems, especially in the neotropics. However, the relationship between ants and non myrmecochorus diaspores is sparsely documented for environments of South America. Thus, this study aimed to quantify the removal rate of non myrmecochorus diaspores by ants in a Cerrado remnant and the influence of energy source presence or its abundance in diaspores for ant removal chance. Ant fauna removed 19.81\% of non myrmecochorus diaspores collected and the presence of energy resources determines their removal, not being a significant relationship between abundance and removal. Since most diaspores collected were not fleshy, with few or none available energy resources for ants, the importance of ant fauna for diaspores removal of non myrmecochocorus may have been underestimated.
\end{abstract}

Keywords: Cerrado, Myrmecochory and Elaiosome.

\section{INTRODUÇÃO}

Depois dos vertebrados, as formigas são consideradas os principais dispersores de sementes em ecossistemas terrestres, esse processo é denominado mirmecocoria (STILES, 1980). E pode rearranjar a chuva de sementes, aumentar a taxa de germinação e influenciar a dinâmica do banco de sementes (PASSOS e OLIVEIRA, 2004).

Como nem sempre a semente é a unidade dispersa, utiliza-se o termo diásporo, que designa a unidade de dispersão. As formigas podem recolher diásporos para sua alimentação, utilizando normalmente o elaiosomo, estrutura atrativa que caracteriza espécies vegetais mirmecocóricas, adaptadas para a dispersão por formigas. A dispersão de diásporos de plantas mirmecocóricas é denominada mirmecocoria verdadeira (MILESI e CASENAVE, 2004).

A relação entre plantas mirmecocóricas e formigas é estudada há pelo menos duas décadas (HANDEL e BEATTIE, 1990). Muitos dos estudos sobre essas interações, entretanto, envolvem apenas uma ou algumas

\footnotetext{
${ }^{1}$ Recebido em 01.10.2008 e aceito para publicação em 25.08.2010.

${ }^{2}$ Universidade do Estado de Minas Gerais, UEMG, Brasil. E-mail: <rbfcampos@gmail.com>, <fernandafarnese@yahoo.com.br> e < gustavofonsecabio@yahoo.com.br>.
} 
poucas espécies de formigas e plantas mirmecocóricas (CASAZZA et al., 2008; ESPADALER e GÓMEZ, 1997; GRESSLER et al., 2006; PIZO e OLIVEIRA, 1998; WHITFORD et al., 2008). Tal fato pode dificultar a visualização da importância dessas interações em condições naturais, em que comunidades são constituídas por inúmeras espécies de formigas e plantas que coexistem e interagem.

Interações entre formigas e plantas não mirmecocóricas (cujas sementes não estão adaptadas para a dispersão por formigas) são pouco documentadas. No entanto, diásporos não mirmecocóricos podem vir a ser dispersos por formigas (MILESI e CASENAVE, 2004; PASSOS e OLIVEIRA, 2003), que podem atuar como dispersoras secundárias (RETANA et al., 2004). A importância das formigas como dispersoras de espécies não mirmecocóricas tem-se tornado evidente (LEAL e OLIVEIRA, 1998; OLIVEIRA et al., 1995; PIZO e OLIVEIRA, 1998). Sabe-se que a mirmecocoria verdadeira não é mecanismo comum de dispersão de sementes em ecossistemas da América do Sul (COSTA et al., 2007), o que ressalta a importância dos estudos com diásporos não mirmecocóricos.

As formigas escolhem os diásporos mirmecocóricos que removem, sendo a presença do elaiosomo o principal fator envolvido nessa escolha (HANDEL e BEATTIE, 1990). Embora a disponibilidade de outros recursos energéticos como a polpa de frutos possa ser importante na Caatinga (LEAL, 2003) e Floresta Atlântica Nordestina (COSTA et al., 2007), não foram encontrados estudos que demonstrem se diásporos não mirmecocóricos são selecionados de alguma forma pelas formigas do Cerrado.

Em trabalho com espécies mirmecocóricas em deserto, Wilby e Shachak (2000) constataram que formigas selecionam as espécies de sementes que coletam e tendem a ser mais seletivas quanto maior a disponibilidade de alimento. Também, podem responder a padrões espaciais de distribuição de alimento, concentrando sua atividade em áreas com grande disponibilidade de sementes. Dessa forma, a abundância de diásporos pode ser fator determinante para a sua dispersão.

Este artigo aborda a relação entre formigas e diásporos não mirmecocóricos em ambiente natural, com o objetivo de quantificar a remoção de diásporos não mirmecocóricos por formigas em ambiente de Cerrado e testar a hipótese de que formigas removem com maior frequência diásporos mais abundantes. A hipótese alternativa é de que formigas selecionam o tipo de diásporo a ser disperso de acordo com a disponibilidade de recursos energéticos.

\section{MATERIAL E MÉTODOS}

\section{1. Área de estudo}

Este estudo foi realizado em fragmento florestal (Parque do Gafanhoto - 2008'21'' S - 4453'17'’ W) localizado no Município de Divinópolis, situado às margens da Rodovia MG 050, na altura da ponte sobre o rio Pará, divisa com o Município de Carmo do Cajuru. O Parque apresenta 19,2 ha, sendo composto, principalmente, por espécies vegetais de Cerrado, não tendo sido registradas, até o momento, espécies vegetais mirmecocóricas em seu interior. O Município de Divinópolis apresenta vegetação típica de Cerrado Sensu Stricto, embora no parque a vegetação se encontre em processo de sucessão secundária. Além disso, espécies exóticas também podem ser encontradas na área.

\subsection{Desenho amostral}

Foram demarcados dois transectos de $100 \mathrm{~m}$ em áreas distintas do Parque do Gafanhoto (Figura 1). Em cada transecto foram marcados cinco pontos, onde foram instalados coletores de sementes distantes 20 $\mathrm{m}$ uns dos outros, totalizando cinco pontos por transecto.

Em cada ponto foi instalado um coletor de sementes. Cada coletor consistiu de quatro estacas de madeira de aproximadamente $50 \mathrm{~cm}$ de altura. As estacas foram fixadas ao solo distantes uma da outra, de modo a formar

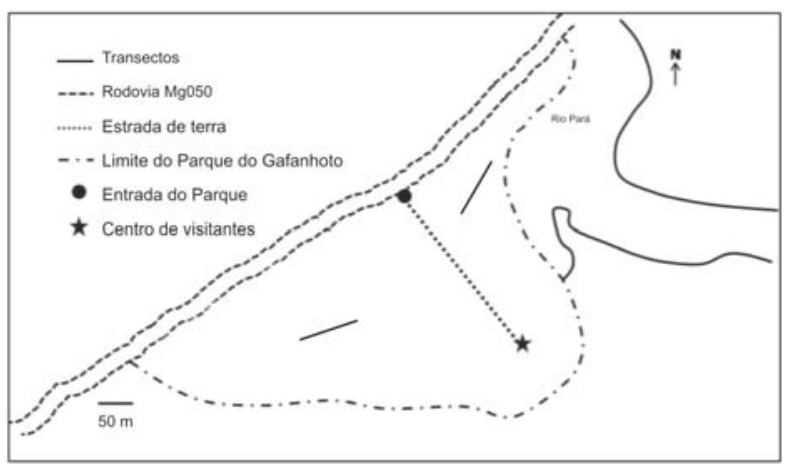

Figura 1 - Localização dos transectos no Parque do Gafanhoto, Divinópolis, MG.

Figure 1 - Location of transects at the Parque do Gafanhoto, Divinópolis, $M G$. 
um quadrado de $1 \mathrm{~m}$ de lado. Um quadrado de tecido não tecido (TNT) foi fixado sobre as estacas como lençol suspenso, tomando-se o cuidado de assegurar que o mesmo ficasse com formato côncavo, a fim de receber os diásporos que caíam da vegetação. A parte da estaca que ficava acima do solo recebeu camada de graxa para impedir o acesso de formigas aos diásporos.

Os coletores permaneceram em campo por sete dias, por campanha de amostragem. Após esse intervalo de tempo, todos os diásporos coletados eram retirados e dispostos em estações de observação. Os diásporos provenientes de um mesmo coletor foram colocados em uma mesma estação, a qual foi instalada no ponto do transecto onde se encontrava o coletor. Cada estação constituía-se de potes plásticos com frestas de aproximadamente $8 \mathrm{~mm}$, que permitiam que as formigas entrassem, manipulassem e removessem os diásporos, mas impediam que animais maiores, como roedores e aves, tivessem acesso a eles.

As estações de observação permaneceram no campo por $48 \mathrm{~h}$, sendo revisadas depois de 12, 24 e $48 \mathrm{~h}$. Durante cada revisão foi registrada a quantidade de diásporos removidos, bem como observado se havia ou não alguma formiga interagindo com eles. Tal procedimento foi repetido uma vez por mês, durante seis meses, sendo três na estação seca (maio, junho e julho) e três na estação chuvosa (outubro, novembro e dezembro), possibilitando, assim, a coleta de diásporos de plantas que se reproduzem em diferentes condições ambientais (MANTOVANI e MARTINS,1988).

Todos os diásporos coletados foram agrupados em morfotipos, segundo suas características morfológicas (presença ou ausência de elaiosomo, presença ou ausência de polpa, porcentagem de polpa e tamanho do diásporo). Os morfotipos foram divididos em dois grandes grupos, carnosos ou secos, considerando-se apenas a presença ou não de polpa. Neste trabalho, o termo “carnoso" foi utilizado para definir os diásporos que apresentavam polpa, enquanto o termo "seco" foi utilizado para se referir àqueles que não apresentavam essa estrutura.

\subsection{Análise de dados: tipo e abundância dos diásporos $\mathrm{x}$ remoção}

Para testar a importância da abundância para a remoção dos diásporos, foi realizada a regressão linear, em que a abundância relativa dos diásporos (número de diásporos de um tipo dividido pelo número total de diásporos nos coletores do transecto em cada coleta) era a variável explicativa e a porcentagem de remoção de cada morfotipo de diásporo, a variável-resposta. O tipo de diásporo foi inserido no modelo como covariável. Utilizou-se a distribuição de erro binominal, sendo posteriormente realizada correção para evitar efeitos de sobredispersão dos dados. O ajuste do modelo foi verificado por meio da análise de distribuição de erros.

Para testar a hipótese de que a presença de recursos energéticos influencia a escolha de diásporos por formigas, realizou-se uma análise de variância, em que a proporção de diásporos removidos foi a variávelresposta, e a presença ou não de polpa (medida de recursos energéticos) era a variável explicativa. Utilizou-se, também, a distribuição de erros binominal, sendo o ajuste do modelo verificado através da análise de distribuição de erros. Todas as análises foram feitas com o auxílio do software R (R DEVELOPMENT CORE TEAM, 2004).

\section{RESULTADOS}

Foram coletados 308 diásporos, 103 na estação seca e 205 na estação chuvosa, classificados em 25 morfotipos, e nenhum deles continha elaiosomo. Entre os diásporos coletados, 61 foram removidos por formigas (19,81\%), os quais pertenciam a sete morfotipos diferentes. Os tipos predominantes de diásporos removidos foram os morfotipos 4 e 5, ambos apresentando grande porcentagem de polpa e pequeno tamanho. Apenas o morfotipo 7 não apresentava polpa (Tabela 1). No ponto com maior abundância foram coletados 57 diásporos, pertencentes a dois morfotipos. Em um dos pontos não foi coletado nenhum diásporo e em dois pontos, coletado apenas um.

Somente formigas pertencentes aos gêneros Atta e Acromyrmex foram visualizadas interagindo e removendo os diásporos nas estações de observação.

O morfotipo mais abundante foi representado por 52 unidades coletadas na estação seca e não apresentava polpa. Nenhuma unidade do morfotipo mais abundante, entretanto, foi removida, e a relação entre a abundância do diásporo e a sua remoção não foi significativa $\left(p=0,64 ; x^{2}=100\right)$. Entretanto, houve relação positiva (Figura 2) entre a presença de recursos energéticos (polpa (fruto classificado com o carnoso)) e a remoção dos diásporos $\left(\mathrm{p}<0,001 ; \mathrm{x}^{2}=11,77\right)$, embora apenas 21,1\% dos diásporos coletados apresentassem polpa.

Revista Árvore, Viçosa-MG, v.35, n.1, p.125-130, 2011 
Tabela 1 - Principais características dos diásporos removidos.

Table 1 - Main features of removed diaspores.

\begin{tabular}{|c|c|c|c|c|c|c|c|}
\hline & $\begin{array}{c}\text { Presença de } \\
\text { elaiosomo }\end{array}$ & $\begin{array}{l}\text { Presença } \\
\text { de polpa }\end{array}$ & $\begin{array}{c}\text { Porcentagem } \\
\text { de polpa }\end{array}$ & $\begin{array}{l}\text { Tamanho } \\
(\mathrm{mm})\end{array}$ & Classificação & $\begin{array}{c}\text { Estação } \\
\text { de coleta }\end{array}$ & $\begin{array}{c}\text { Taxa relativa } \\
\text { de remoção(\%) }\end{array}$ \\
\hline$\overline{\text { Morfotipo } 1}$ & - & $\mathrm{x}$ & $<50$ & $<5$ & Carnoso & Chuvosa & 16 \\
\hline Morfotipo 2 & - & $\mathrm{x}$ & 100 & $>10$ & Carnoso & Chuvosa & 63 \\
\hline Morfotipo 3 & - & $\mathrm{x}$ & $>50$ & 5 a 10 & Carnoso & Seca & 59 \\
\hline Morfotipo 4 & - & $\mathrm{x}$ & 100 & $<5$ & Carnoso & Chuvosa & 83 \\
\hline Morfotipo 5 & - & $\mathrm{x}$ & 100 & $<5$ & Carnoso & Chuvosa & 100 \\
\hline Morfotipo 6 & - & $\mathrm{x}$ & $<50$ & 5 a 10 & Carnoso & Seca & 27 \\
\hline Morfotipo 7 & - & - & 0 & 5 a 10 & Seco & Seca & 7 \\
\hline
\end{tabular}

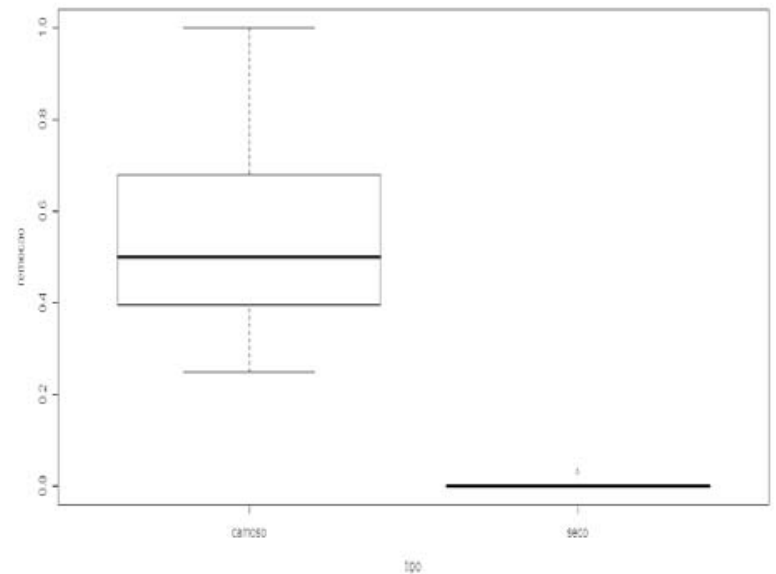

Figura 2 - Tipo de diásporo versus remoção dada em proporção de diásporos removidos por estação de observação $\left(\mathrm{p}<0,001 ; \chi^{2}=11,77\right)$. As barras mostram a proporção média de dispersão por formigas e os desvios para diásporos carnosos (com polpa) e secos (sem polpa) dispostos nas estações, no Parque do Gafanhoto, Divinópolis, MG.

Figure 2-Diaspore type versus removal given as proportion of removed diaspores observational station ( $\left.p<0001 ; \chi^{2}=11.77\right)$. Bars show the average proportion dispersal by ants and deviation to fleshy (with pulp) and dry (without pulp) diaspores arranged in the stations at the Parque do Gafanhoto, Divinópolis, $M G$.

\section{DISCUSSÃO}

A porcentagem de remoção de diásporos encontrada neste trabalho $(19,81 \%)$ não foi tão alta quanto aquela encontrada em outros estudos, nos quais foram utilizados diásporos mirmecocóricos e onde as formigas dispersaram mais da metade dos diásporos (GÓMEZ e OLIVERAS, 2003; PETERNELLI et al., 2004). Assim, aparentemente a remoção de diásporos não mirmecocóricos não é processo tão significativo quanto a dispersão de diásporos mirmecocóricos.

A taxa de remoção de diásporos não mirmecocóricos pode, no entanto, ter sido subestimada, uma vez que apenas 21,1\% dos diásporos coletados possuíam polpa, e esse foi um recurso que influenciou significativamente a taxa de remoção dos diásporos pelas formigas. Se considerar apenas os diásporos com polpa, a porcentagem total de remoção sobe de $19,81 \%$ para $53,84 \%$, maior do que a encontrada por Santo (2007), que constatou que formigas podem remover até $40 \%$ das sementes coletadas de Ricinus communis, uma espécie mirmecocórica. A quantidade de polpa encontrada no fruto, entretanto, não foi testada neste estudo, sendo considerada apenas a sua presença ou ausência.

Apenas formigas dos gêneros Atta e Acromyrmex foram visualizadas removendo os diásporos nas estações de observação. Embora essas formigas sejam consideradas pragas em agroecossistemas e florestas implantadas (BOARETTO e FORTI, 1997), elas já foram observadas promovendo a germinação de sementes em Floresta Atlântica (PIZO e OLIVEIRA, 1998), Semidecídua (OLIVEIRA et al., 1995; PASSOS e FERREIRA, 1996) e Cerrado (LEAL e OLIVEIRA, 1998, 2000). Além disso, já foram relatadas como capazes de dispersarem os diásporos a uma distância maior do que fazem os demais gêneros de formigas, sendo isso, provavelmente, devido ao seu corpo robusto (SANTO, 2007). Formigas desses gêneros apresentam hábitos alimentares generalistas (HÖLLDOBLER e WILSON, 1990), e talvez esse seja o motivo pelo qual apenas elas foram visualizadas interagindo com diásporos não mirmecocóricos, evidenciando que a dispersão de diásporos mirmecocóricos é um processo mais especializado que a dispersão de diásporos não mirmecocóricos. 
Muitas espécies de formigas, como Pheidole, Aphaenogaster e Tapinoma, já foram, entretanto, registradas como dispersoras de sementes, inclusive não mirmecocóricas (COSTAetal., 2007; LEAL, 2003). É possível que, embora não tenham sido visualizadas, formigas de outros grupos generalistas como Camponotus e Pheidole e grupos que apresentam espécies predadoras como Pachycondyla, e Odontomachus, além de outras cultivadoras de fungos da tribo Attini, tenham contribuído para a remoção dos diásporos dispostos nas estações de observação. Essa possibilidade é levantada, uma vez que essas formigas fazem parte da fauna do parque (observação pessoal), e observações nas estações com diásporos eram feitas em intervalos de pelo menos $12 \mathrm{~h}$.

A presença ou não de polpa foi mais importante que a abundância para a remoção dos diásporos. O fato de a relação entre abundância e remoção dos diásporos não ser significativa evidencia que formigas, de alguma forma, escolhem os diásporos a serem por elas removidos, em vez de simplesmente removerem o tipo mais frequente.

A relação positiva entre presença de polpa e remoção de diásporos reforça a inferência de que a presença de recursos energéticos é fundamental para a atratividade de formigas. Possivelmente, a presença de polpa em diásporos não mirmecocóricos tem efeito análogo ao elaiosomo em sementes mirmecocóricas. Outros estudos têm demonstrado a importância da polpa para a atratividade de formigas em ambientes de Caatinga (LEAL, 2003) e Floresta Atlântica Nordestina (COSTA et al., 2007). Nesses trabalhos, no entanto, os diásporos foram dispostos próximos à entrada dos ninhos e, ou, selecionados previamente, o que pode ter interferido no processo de dispersão. Neste artigo, diferentemente do que havia sido feito até então, todos os diásporos presentes nos coletores permaneceram no local, onde foram coletados em estações de observação, o que permite obter informações mais próximas do que acontece naturalmente na área estudada. Além disso, o número de diásporos nos trabalhos citados foi disposto sempre de modo massivo, criando situação diferente da real. Essa concentração de recurso modifica o comportamento das formigas (WILBY e SHACHAK, 2000), podendo alterar o padrão de remoção.

\section{CONCLUSÃO}

Através dos resultados, é possível concluir que a dispersão de diásporos por formigas é um processo importante também para plantas não mirmecocóricas.
Esse processo pode ter importantes implicações, uma vez que outros dispersores, como aves e pequenos mamíferos, são afetados diretamente pela fragmentação decorrente do desmatamento e urbanização, como é o caso do parque estudado e áreas com características semelhantes às deste. Nesses casos, as interações entre formigas e diásporos podem ser ainda mais importantes, e é fundamental compreender as condições em que essas interações ocorrem tanto para a conservação quanto para a recuperação de ambientes (JORDANO et al., 2006). Os resultados também demonstram que as características dos diásporos não mirmecocóricos são mais importantes que a sua abundância da dispersão por formigas.

\section{AGRADECIMENTOS}

Ao Professor José Henrique Schoereder, da Universidade Federal de Viçosa, pelo auxílio nas análises estatísticas; ao Programa de Bolsas PAPq - ISED/FUNEDI/ UEMG, pelo auxílio financeiro; e aos dois revisores anônimos, pelas sugestões referentes à redação do texto.

\section{REFERÊNCIAS}

BOARETTO, M. A. C.; FORTI, L. C. Perspectivas no controle de formigas cortadeiras. Série Técnica IPEF, v.11, n.30, p.31-46, 1997.

CASAZZA, G. et al. Dispersal mechanisms in some representatives of the genus Moehringia L. (Caryophyllaceae). Acta Oecologica, v.33, p.246-252, 2008.

COSTA, U. A. S. et al. Dispersão de sementes por formigas em remanescentes de floresta atlântica nordestina. Revista Brasileira de Biociências, v.5, supl.1, p.231-233, 2007.

ESPADALER, X.; GÓMEZ, C. Soil surface searching and transport of Euphorbia characias seeds by ants. Acta Oecologica, v.18, n.1, p.39-46, 1997.

GÓMEZ, C.; OLIVERAS, J. Can the Argentine ant (Linepithema humile Mayr) replace native ants in myrmecochory? Acta Oecologica, v.24, n.1, p.47-53, 2003.

GRESSLER, E.; PIZO, M. A.; MORELLATO, P. C. Polinização e dispersão de sementes em Myrtaceae do Brasil. Revista Brasileira de Botância, v.29, n.4, p.509-530, 2006.

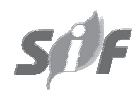

Revista Árvore, Viçosa-MG, v.35, n.1, p.125-130, 2011 
HANDEL, S. N.; BEATTIE, A. J. Seed dispersal by ants. Science American, v.263, n.1, p.54-61, 1990.

HÖLlDOBLER, B.; WILSON, E. O. The Ants. Cambridge: Harvard University Press, 1990. 76p.

JORDANO, P. et al. Ligando Frugivoria e dispersão de sementes à biologia da conservação. In: DUARTE, C. F. et al. Biologia da conservação: essências. São Paulo: Rima, 2006. p.411-436.

LEAL, I. R. Dispersão de sementes por formigas na caatinga. In: LEAL, I. R.; TABARELLI, M.; SILVA, J. M. C. (Eds.). Ecologia e conservação da caatinga. Recife: Universitária UFPE, 2003. p.593-624.

LEAL, I. R.; OLIVEIRA, P. S. Foraging ecology of attine ants in a Neotropical savanna: seasonal use of fungal substrate in the cerrado vegetation o Brazil. Insectes Sociaux, v.47, p.376-382, 2000.

LEAL, I. R.; OLIVEIRA, P. S. Interactions between fungus-growing ants (Attini), fruits and seeds in Cerrado vegetation in southeast Brazil.

Biotropica, v.30, p.170-178, 1998.

MANTOVANI, W.; MARTINS, F. R. Variações fenológicas das espécies do cerrado na Reseva Biológica de Moji-Guaçu, estado de São Paulo. Revista Brasileira de Botânica, v.11, p.101-112, 1988.

MILESI, F. A.; CASENAVE, J. L. Unexpected relationships and valuable mistakes: nonmyrmecochorous Prosopis dispersed by messy leafcutting ants in harvesting their seeds. Austral Ecology, v.29, p.558-567, 2004.

OLIVEIRA, P. S. et al. Seed cleaning by Mycocepurus goeldii ants (Attini) facilitates germination in Hymenaea courbaril (Caesalpiniaceae).

Biotropica, v.27, p.518-522, 1995.

PASSOS, L.; FERREIRA, S. O. Ant dispersal of Croton priscus (Euphorbiaceae) seeds in a tropical semideciduous forest in Souteastern Brazil. Biotropica, v.28, p.697-700, 1996.

PASSOS, L.; OLIVEIRA, P. S. Interactions between ants, fruits and seeds in a restinga forest in south-eastern Brazil. Journal of Tropical Ecology, v.19, p.261-270, 2003.
PASSOS, L.; OLIVEIRA, P. S. Interaction between ants and fruits of Guapira opposita (Nyctaginaceae) in a Brazilian sandy plain rainforest: ant effects on seeds and seedlings. Oecologia, v.139, n.3, p.376-382, 2004.

PETERNELLI, E. F. O.; DELLA LUCIA, T. M. C.; MARTINS, S. V. Espécies de formigas que interagem com as sementes de Mabea fistulifera Mart. (Euphorbiaceae). Revista Árvore, v.28, n.5, p.733-738, 2004.

PIZO, M. A.; OLIVEIRA, P. S. Interaction between ants and seeds of a nonmyrmechocorous neotropical tree, Cabralea canjerana (Meliaceae), in the Atlantic forest of Southeast Brazil. American Journal of Botany, v.85, p.669-674, 1998.

R DEVELOPMENT CORE TEAM. 2004. R: A

language and environment for statistical computing. Vienna: R Foundation for Statistical Computing, 2004. URL http://www.R-project.org.

RETANA, J.; PICÓ, F. X.; RODRIGO, A. Dual role of harvesting ants as seeds predators and disperses of a non-myrmechorous Mediterrenean perennial herb. Oikos, v.105, p.377-385, 2004.

SANTO, M. M. E. Secondary seed dispersal of Ricinus communis Linnaeus (Euphorbiaceae) by ants in secondary growth vegetation in Minas gerais. Revista Árvore, v.31, n.6, p.1013-1018, 2007.

STILES, E. W. Patterns of fruit presentation and seed dispersal in bird-disseminated woody plants in the earsten deciduous forest. The American Naturalist, v.116, n.5, p.670-688, 1980.

WHITFORD, W. G.; BARNESS, G.; STEINBERGER, Y. Effects of three species of Chihuahuan Desert ants on annual plants and soil properties.

Journal of Arid Environments, v.72, p.392-400, 2008.

WILBY, A.; SHACHAK, M. Harvester ant response to spatial and temporal heterogeneity in seed availability: pattern in the process of granivory. Oecologica, v.125, p.495-503, 2000. 